Conclusions This study suggests us that monitoring of recall behaviour would be an efficient and practical tool for compliance assessment of players in the market. It can tell us which manufacturers are possibly very reluctant or lazy in recall actions. Also this study suggest us some improvement is needed for accident database and recall database.

\section{EPIDEMIOLOGICAL CHARACTERISTICS OF PRODUCT HARM CASES IN 32 HOSPITALS IN 11 AREAS IN CHINA, 2012-2014}

Ye Pengpeng, Er Yuliang, Wang Yuan, Deng Xiao, Duan Leilei. National Centre for Chronic and Noncommunicable Disease Control and Prevention, Chinese Centre for Disease Control and Prevention, China

\subsection{6/injuryprev-2016-042156.810}

Background To understand the epidemiological characteristics of product harm cases in 32 hospitals in 11 areas in China from 2012 to 2014 and provide the basic data to support for productspecific survey, product harm early warning and the assessment of product safety.

Methods The descriptive epidemiologic analysis was conducted by using the surveillance data of product harm collected from 32 hospitals in 11 areas in China during 2012-2014.

Results A total of 208772 product harm cases were reported in the 32 hospitals during 2012-2014, accounting for $19.50 \%$ of total harm cases during the same period. A total of 222401 cases (times) of product harm were reported. For all the product harms, the top five products causing harms were transportation equipment except motor vehicle (36.55\%), motor vehicle (21.50\%), other products (20.84\%), furniture (7.21\%) and food, medicine and related products $(5.18 \%)$. Both the case number and times of product harm were higher in males than in females. Males aged 25-44 years might be at greater risk for product harm. Most product harms, i.e. bruise, were caused by blunt force on heads.

Conclusions The epidemiologic characteristics of product harm varied with products. It is necessary to take targeted intervention measures to prevent product harm in China.

\section{THE USE OF INJURY DATA AS DRIVER FOR EFFECTIVE PRODUCT SAFETY STANDARDS}

Tania Vandenberghe. ANEC, the European Consumer Voice in Standardisation, Brussels

\subsection{6/injuryprev-2016-042156.811}

Background For consumers, standards are important. When they are properly developed and applied, they can make our lives easier, the products we buy safer, and help prevent injuries and accidents. In order to properly develop standards leading to safer products, it is very important to have accident and injury statistics at our disposal.

Methods One powerful aspect of ANEC's pan-European network of experts is that it has the ability to spot emerging trends, as there is no pan-European data system that allows this to happen. Thanks to the availability of accident data from some countries, ANEC asked the European Standardisation organisation CEN for standards to be set up for cords and drawstrings on children's clothing, moveable goals and 'nappy sacks', and asked for improving the standards for cigarette lighters and window blinds.
Results ANEC was successful in asking for standards to be set up for cords and drawstrings on children's clothing, for moveable goals, and in improving the safety of cigarette lighters and window blinds through standardisation. Unfortunately, only a few countries within the EU systematically collect accident and injury data. This lack of data is often used by some stakeholders as an excuse not to improve standards. As a result, ANEC has not been able for the standard for small off-road motorbikes to address child safety aspects, nor for the standard for the safety of children's clothing to address the risk of hoods. The request from ANEC to set up a standard for 'nappy sacks' was not followed up, as data on fatal accidents was only available from one country.

Conclusions Lack of EU-funded accident \& injury data system makes it difficult to assess where legislative, standardisation, market surveillance, and/or awareness raising actions are needed and to assess the success of measures/actions taken. Therefore, there is an urgent need for a pan-European accident $\&$ injury data system, funded by the European Commission.

\section{REVISION OF THE SAFETY STANDARDS FOR A BABY CHAIR UTILISING INJURY DATA}

\begin{abstract}
1,2,3 Tatsuhiro Yamanaka, 2,3Yoshifumi Nishida, 2,3Mikiko Oono, 2,3 Koji Kitamura, ${ }^{4}$ Takeshi Sato, ${ }^{4}$ Shuichi Kurokawa. 'Ryokuen Children's Clinic, Japan; ${ }^{2}$ National Institute of Advanced Industrial Science and Technology, Japan; ${ }^{3}$ Safe Kids Japan, Japan; ${ }^{4}$ Consumer Product Safety Association, Japan
\end{abstract}

\subsection{6/injuryprev-2016-042156.812}

Background Since the SG (Safe Goods) Standards for a baby chair was enacted by Consumer Product Safety Association in 1981, no revision has been made for 30 years. During these years, new types of products to which the SG Standards did not apply became available, serious injuries related to old type chairs were reported, and other issues came up related to a baby chair. Then it is necessary to revise the standard as soon as possible. The purpose of this study is to analyse baby chair-related injury data and revise the safety quality requirements.

Methods We analysed 99 baby chair-related injuries that required ambulance transport to identify a specific body part being caught and a part of a chair that caused injury in cooperation with Tokyo Fire Department.

Results The body parts caught in a baby pipe chair, a metal-pipeframe low chair which is usually cheap with simple design, were: fingers (31 cases), feet (18 cases), head/neck (10 cases), toes (8 cases), and ankles (4 cases). Among 99 cases, 10 cases occurred in 0 year old, 28 cases in 1 year old, 26 cases in 2 years old, 13 cases in 3 years old, 14 cases in 4 years old, and 5 cases in 5 years old. When a child's body size is within 25 percentiles, his or her head is bigger than a trunk. Thus, in order to check the entrapment and strangulation hazard, we developed a probe $(95 \times 140 \mathrm{~mm})$ which mimics a trunk of a child body utilising data on a body trunk jig dimension suited for Japanese infants of National Institute of Technology and Evaluation. The revised standard requires that we must use the developed probe to check injury risk.

Conclusions As a result of injury data analysis, we found that children under 2 were more prone to a baby chair-related injury and understood how injury occurred in details. When a child's head is caught in clearance between the parts of a baby chair, it might cause suffocation. We developed a trunk probe to check injury risk for a pipe chair for ages 7-36 months and established a new safety standard requirement. From this standard revision 